\title{
Search for baryon and lepton number violating decays $D^{+} \rightarrow \bar{\Lambda}\left(\overline{\boldsymbol{\Sigma}}^{0}\right) e^{+}$and $D^{+} \rightarrow \Lambda\left(\Sigma^{0}\right) e^{+}$
}

M. Ablikim, ${ }^{1}$ M. N. Achasov,${ }^{10, *}$ P. Adlarson,${ }^{59}$ S. Ahmed ${ }^{15}$ M. Albrecht,${ }^{4}$ M. Alekseev,${ }^{58 a, 58 c}$ A. Amoroso, ${ }^{58 a, 58 c}$ F. F. An, ${ }^{1}$ Q. An ${ }^{55,43}$ Y. Bai, ${ }^{42}$ O. Bakina, ${ }^{27}$ R. Baldini Ferroli, ${ }^{23 a}$ I. Balossino, ${ }^{24 a}$ Y. Ban, ${ }^{35,}$ K. Begzsuren, ${ }^{25}$ J. V. Bennett, ${ }^{5}$

N. Berger, ${ }^{26}$ M. Bertani, ${ }^{23 a}$ D. Bettoni, ${ }^{24 a}$ F. Bianchi, ${ }^{58 a, 58 c}$ J. Biernat, ${ }^{59}$ J. Bloms,${ }^{52}$ I. Boyko, ${ }^{27}$ R. A. Briere,${ }^{5}$ H. Cai, ${ }^{60}$ X. Cai, ${ }^{1,43}$ A. Calcaterra, ${ }^{23 a}$ G. F. Cao, ${ }^{1,47}$ N. Cao,${ }^{1,47}$ S. A. Cetin, ${ }^{46 b}$ J. Chai ${ }^{58 c}$ J. F. Chang, ${ }^{1,43}$ W. L. Chang, ${ }^{1,47}$ G. Chelkov, ${ }^{27}{ }^{\ddagger}, 8$ D. Y. Chen, ${ }^{6}$ G. Chen, ${ }^{1}$ H. S. Chen, ${ }^{1,47}$ J. C. Chen, ${ }^{1}$ M. L. Chen, ${ }^{1,43}$ S. J. Chen, ${ }^{33}$ Y. B. Chen, ${ }^{1,43}$ W. Cheng, ${ }^{58 \mathrm{c}}$ G. Cibinetto ${ }^{24 \mathrm{a}}$ F. Cossio, ${ }^{58 \mathrm{c}}$ X. F. Cui, ${ }^{34}$ H. L. Dai, ${ }^{1,43}$ J. P. Dai,${ }^{38, \|}$ X. C. Dai, ${ }^{1,47}$ A. Dbeyssi,${ }^{15}$ D. Dedovich ${ }^{27}$ Z. Y. Deng, ${ }^{1}$ A. Denig ${ }^{26}$ I. Denysenko, ${ }^{27}$ M. Destefanis, ${ }^{58 a, 58 c}$ F. De Mori ${ }^{58 a, 58 c}$ Y. Ding ${ }^{31}$ C. Dong, ${ }^{34}$ J. Dong, ${ }^{1,43}$ L. Y. Dong, ${ }^{1,47}$ M. Y. Dong, ${ }^{1,43,47}$ Z. L. Dou ${ }^{33}$ S. X. Du, ${ }^{63}$ J. Z. Fan, ${ }^{45}$ J. Fang, ${ }^{1,43}$ S. S. Fang, ${ }^{1,47}$ Y. Fang, ${ }^{1}$ R. Farinelli, ${ }^{24 a, 24 b}$ L. Fava,${ }^{58 b, 58 c}$ F. Feldbauer, ${ }^{4}$ G. Felici, ${ }^{23 a}$ C. Q. Feng, ${ }^{55,43}$ M. Fritsch, ${ }^{4}$ C. D. Fu, ${ }^{1}$ Y. Fu, ${ }^{1}$ Q. Gao, ${ }^{1}$ X. L. Gao, ${ }^{55,43}$ Y. Gao, ${ }^{56}$ Y. Gao, ${ }^{45}$ Y. G. Gao, ${ }^{6}$ Z. Gao, ${ }^{55,43}$ B. Garillon, ${ }^{26}$ I. Garzia ${ }^{24 a}$ E. M. Gersabeck,${ }^{50}$ A. Gilman ${ }^{51}$ K. Goetzen, ${ }^{11}$ L. Gong, ${ }^{34}$ W. X. Gong, ${ }^{1,43}$ W. Gradl, ${ }^{26}$ M. Greco, ${ }^{58 a, 58 c}$ L. M. Gu, ${ }^{33}$ M. H. Gu, ${ }^{1,43}$ S. Gu, ${ }^{2}$ Y. T. Gu, ${ }^{13}$ A. Q. Guo, ${ }^{22}$ L. B. Guo, ${ }^{32}$ R. P. Guo, ${ }^{36}$ Y. P. Guo, ${ }^{26}$ A. Guskov, ${ }^{27}$ S. Han ${ }^{60}$ X. Q. Hao, ${ }^{16}$ F. A. Harris, ${ }^{48}$ K. L. He, ${ }^{1,47}$ F. H. Heinsius, ${ }^{4}$ T. Held, ${ }^{4}$ Y. K. Heng, ${ }^{1,43,47}$ M. Himmelreich, ${ }^{11,}$ Y. R. Hou, ${ }^{47}$ Z. L. Hou, ${ }^{1}$ H. M. Hu, ${ }^{1,47}$ J. F. Hu, ${ }^{38, \|}$ T. Hu, ${ }^{1,43,47}$ Y. Hu, ${ }^{1}$ G. S. Huang, ${ }^{55,43}$ J. S. Huang, ${ }^{16}$ X. T. Huang ${ }^{37}$ X. Z. Huang ${ }^{33}$ N. Huesken, ${ }^{52}$ T. Hussain, ${ }^{57}$ W. Ikegami Andersson, ${ }^{59}$ W. Imoehl, ${ }^{22}$ M. Irshad, ${ }^{55,43}$ Q. Ji, ${ }^{1}$ Q. P. Ji, ${ }^{16}$ X. B. Ji ${ }^{1,47}$ X. L. Ji, ${ }^{1,43}$ H. L. Jiang, ${ }^{37}$ X. S. Jiang, ${ }^{1,43,47}$ X. Y. Jiang, ${ }^{34}$ J. B. Jiao, ${ }^{37}$ Z. Jiao, ${ }^{18}$ D. P. Jin, ${ }^{1,43,47}$ S. Jin ${ }^{33}$ Y. Jin ${ }^{49}$ T. Johansson, ${ }^{59}$ N. Kalantar-Nayestanaki, ${ }^{29}$ X. S. Kang, ${ }^{31}$

R. Kappert, ${ }^{29}$ M. Kavatsyuk, ${ }^{29}$ B. C. Ke, ${ }^{1}$ I. K. Keshk, ${ }^{4}$ A. Khoukaz, ${ }^{52}$ P. Kiese, ${ }^{26}$ R. Kiuchi, ${ }^{1}$ R. Kliemt, ${ }^{11}$ L. Koch, ${ }^{28}$ O. B. Kolcu, ${ }^{46 b,{ }^{* *}}$ B. Kopf ${ }^{4}$ M. Kuemmel, ${ }^{4}$ M. Kuessner, ${ }^{4}$ A. Kupsc, ${ }^{59}$ M. Kurth, ${ }^{1}$ M. G. Kurth, ${ }^{1,47}$ W. Kühn, ${ }^{28}$ J. S. Lange, ${ }^{28}$ P. Larin, ${ }^{15}$ L. Lavezzi, ${ }^{58 c}$ H. Leithoff, ${ }^{26}$ T. Lenz,${ }^{26}$ C. Li ${ }^{59}$ Cheng Li,${ }^{55,43}$ D. M. Li ${ }^{63}$ F. Li,${ }^{1,43}$ F. Y. Li, ${ }^{35, \dagger}$ G. Li, ${ }^{1}$ H. B. Li, ${ }^{1,47}$ H. J. Li, ${ }^{9}{ }^{\dagger \dagger}$ J. C. Li, ${ }^{1}$ J. W. Li,${ }^{41}$ Ke Li,${ }^{1}$ L. K. Li, ${ }^{1}$ Lei Li,${ }^{3}$ P. L. Li ${ }^{55,43}{ }^{2}$ P. R. Li,${ }^{30}$ Q. Y. Li,${ }^{37}$ W. D. Li, ${ }^{1,47}$ W. G. Li, ${ }^{1}$ X. H. Li ${ }^{55,43}$ X. L. Li,${ }^{37}$ X. N. Li, ${ }^{1,43}$ Z. B. Li, ${ }^{44}$ Z. Y. Li, ${ }^{44}$ H. Liang,${ }^{55,43}$ H. Liang, ${ }^{1,47}$ Y. F. Liang, ${ }^{40}$ Y. T. Liang ${ }^{28}$ G. R. Liao, ${ }^{12}$ L. Z. Liao, ${ }^{1,47}$ J. Libby, ${ }^{21}$ C. X. Lin, ${ }^{44}$ D. X. Lin ${ }^{15}$ Y. J. Lin, ${ }^{13}$ B. Liu, ${ }^{38, \|}$ B. J. Liu, ${ }^{1}$ C. X. Liu, ${ }^{1}$ D. Liu, ${ }^{5,43}$ D. Y. Liu, ${ }^{38, \|}$ F. H. Liu, ${ }^{39}$ Fang Liu, ${ }^{1}$ Feng Liu, ${ }^{6}$ H. B. Liu, ${ }^{13}$ H. M. Liu ${ }^{1,47}$ Huanhuan Liu, ${ }^{1}$ Huihui Liu, ${ }^{17}$ J. B. Liu, ${ }^{55,43}$ J. Y. Liu, ${ }^{1,47}$ K. Y. Liu, ${ }^{31}$ Ke Liu, ${ }^{6}$ L. Y. Liu, ${ }^{13}$ Q. Liu, ${ }^{47}$ S. B. Liu, ${ }^{55,43}$ T. Liu, ${ }^{1,47}$ X. Liu, ${ }^{30}$ X. Y. Liu,,${ }^{1,47}$ Y. B. Liu, ${ }^{34}$ Z. A. Liu, ${ }^{1,43,47}$ Zhiqing Liu, ${ }^{37}$ Y. F. Long, ${ }^{35,}{ }^{+}$X. C. Lou, ${ }^{1,43,47}$ H. J. Lu, ${ }^{18}$ J. D. Lu, ${ }^{1,47}$ J. G. Lu, ${ }^{1,43}$ Y. Lu, ${ }^{1}$ Y. P. Lu, ${ }^{1,43}$ C. L. Luo, ${ }^{32}$ M. X. Luo, ${ }^{62}$ P. W. Luo, ${ }^{44}$ T. Luo, ${ }^{9, \dagger}$ X. L. Luo, ${ }^{1,43}$ S. Lusso, ${ }^{58 c}$ X. R. Lyu, ${ }^{47}$ F. C. Ma, ${ }^{31}$ H. L. Ma, ${ }^{1}$ L. L. Ma, ${ }^{37}$ M. M. Ma ${ }^{1,47}$ Q. M. Ma, ${ }^{1}$ X. N. Ma,${ }^{34}$ X.X. Ma,${ }^{1,47}$ X. Y. Ma ${ }^{1,43}$ Y. M. Ma ${ }^{37}$ F. E. Maas, ${ }^{15}$ M. Maggiora, ${ }^{58 a, 58 c}$ S. Maldaner, ${ }^{26}$ S. Malde ${ }^{53}$ Q. A. Malik, ${ }^{57}$ A. Mangoni ${ }^{23 b}$ Y. J. Mao, ${ }^{35,}$ Z. P. Mao, ${ }^{1}$ S. Marcello ${ }^{58 a, 58 c}$ Z. X. Meng, ${ }^{49}$ J. G. Messchendorp, ${ }^{29}$ G. Mezzadri, ${ }^{24 a}$ J. Min, ${ }^{1,43}$ T. J. Min ${ }^{33}$ R. E. Mitchell, ${ }^{22}$ X. H. Mo, ${ }^{1,43,47}$ Y. J. Mo, ${ }^{6}$

C. Morales Morales, ${ }^{15}$ N. Yu. Muchnoi,${ }^{10,{ }^{*}}$ H. Muramatsu, ${ }^{51}$ A. Mustafa,${ }^{4}$ S. Nakhoul, ${ }^{11,}$ Y. Nefedov,${ }^{27}$ F. Nerling, ${ }^{11,}$ I. B. Nikolaev, ${ }^{10, *}$ Z. Ning, ${ }^{1,43}$ S. Nisar, ${ }^{8, *}$ S. L. Niu, ${ }^{1,43}$ S. L. Olsen, ${ }^{47}$ Q. Ouyang, ${ }^{1,43,47}$ S. Pacetti, ${ }^{23 b}$ Y. Pan, ${ }^{55,43}$ M. Papenbrock, ${ }^{59}$ P. Patteri, ${ }^{23 a}$ M. Pelizaeus ${ }^{4}$ H. P. Peng,${ }^{55,43}$ K. Peters, ${ }^{11,}$ J. Pettersson, ${ }^{59}$ J. L. Ping, ${ }^{32}$ R. G. Ping, ${ }^{1,47}$ A. Pitka, ${ }^{4}$ R. Poling, ${ }^{51}$ V. Prasad,${ }^{55,43}$ H. R. Qi ${ }^{2}$ M. Qi ${ }^{33}$ T. Y. Qi ${ }^{2}$ S. Qian, ${ }^{1,43}$ C. F. Qiao, ${ }^{47}$ N. Qin, ${ }^{60}$ X. P. Qin, ${ }^{13}$ X. S. Qin, ${ }^{4}$ Z. H. Qin, ${ }^{1,43}$ J. F. Qiu, ${ }^{1}$ S. Q. Qu ${ }^{34}$ K. H. Rashid, ${ }^{57, \$ 8}$ K. Ravindran, ${ }^{21}$ C. F. Redmer, ${ }^{26}$ M. Richter, ${ }^{4}$ A. Rivetti, ${ }^{58 c}$ V. Rodin, ${ }^{29}$ M. Rolo ${ }^{58 c}$ G. Rong, ${ }^{1,47}$ Ch. Rosner, ${ }^{15}$ M. Rump, ${ }^{52}$ A. Sarantsev, ${ }^{27, \|}$. M. Savrié, ${ }^{24 b}$ Y. Schelhaas,${ }^{26}$ K. Schoenning, ${ }^{59}$ W. Shan, ${ }^{19}$ X. Y. Shan, ${ }^{55,43}$ M. Shao, ${ }^{55,43}$ C. P. Shen, ${ }^{2}$ P. X. Shen ${ }^{34}$ X. Y. Shen,${ }^{1,47}$ H. Y. Sheng, ${ }^{1}$ X. Shi, ${ }^{1,43}$ X. D. Shi ${ }^{55,43}$ J. J. Song, ${ }^{37}$ Q. Q. Song,${ }^{5,43}$ X. Y. Song, ${ }^{1}$ S. Sosio, ${ }^{58 a, 58 c}$ C. Sowa ${ }^{4}$ S. Spataro, ${ }^{58 a, 58 c}$ F. F. Sui, ${ }^{37}$ G. X. Sun, ${ }^{1}$ J. F. Sun, ${ }^{16}$ L. Sun, ${ }^{60}$ S. S. Sun, ${ }^{1,4}$ X. H. Sun, ${ }^{1}$ Y. J. Sun,${ }^{55,43}$ Y. K. Sun,${ }^{55,43}$ Y. Z. Sun, ${ }^{1}$ Z. J. Sun, ${ }^{1,43}$ Z. T. Sun, ${ }^{1}$ Y. T. Tan,${ }^{55,43}$ C. J. Tang, ${ }^{40}$ G. Y. Tang, ${ }^{1}$ X. Tang, ${ }^{1}$ V. Thoren, ${ }^{59}$ B. Tsednee, ${ }^{25}$ I. Uman, ${ }^{46 d}$ B. Wang, ${ }^{1}$ B. L. Wang, ${ }^{47}$ C. W. Wang, ${ }^{33}$ D. Y. Wang, ${ }^{3, \dagger}$ K. Wang, ${ }^{1,43}$ L. L. Wang, ${ }^{1}$ L. S. Wang, ${ }^{1}$ M. Wang, ${ }^{37}$ M. Z. Wang, ${ }^{35,{ }^{*}}$ Meng Wang, ${ }^{1,47}$

P. L. Wang, ${ }^{1}$ R. M. Wang, ${ }^{61}$ W. P. Wang ${ }^{55,43}$ X. Wang ${ }^{35, \dagger}$ X. F. Wang, ${ }^{1}$ X. L. Wang, ${ }^{9, \dagger}$ Y. Wang, ${ }^{44}$ Y. Wang, ${ }^{55,43}$ Y. F. Wang, ${ }^{1,43,47}$ Y. Q. Wang, ${ }^{1}$ Z. Wang, ${ }^{1,43}$ Z. G. Wang, ${ }^{1,43}$ Z. Y. Wang, ${ }^{1}$ Zongyuan Wang, ${ }^{1,47}$ T. Weber, ${ }^{4}$ D. H. Wei, ${ }^{12}$ P. Weidenkaff, ${ }^{26}$ H. W. Wen, ${ }^{32}$ S. P. Wen, ${ }^{1}$ U. Wiedner, ${ }^{4}$ G. Wilkinson, ${ }^{53}$ M. Wolke, ${ }^{59}$ L. H. Wu, ${ }^{1}$ L. J. Wu, ${ }^{1,47}$ Z. Wu,${ }^{1,43}$ L. Xia, ${ }^{55,43}$ Y. Xia, ${ }^{20}$ S. Y. Xiao, ${ }^{1}$ Y. J. Xiao, ${ }^{1,47}$ Z. J. Xiao, ${ }^{32}$ Y. G. Xie, ${ }^{1,43}$ Y. H. Xie, ${ }^{6}$ T. Y. Xing,${ }^{1,47}$ X. A. Xiong,,${ }^{1,47}$ Q. L. Xiu, ${ }^{1,43}$ G. F. Xu, ${ }^{1}$ J. J. Xu ${ }^{33}$ L. Xu, ${ }^{1}$ Q. J. Xu, ${ }^{14}$ W. Xu,${ }^{1,47}$ X. P. Xu ${ }^{41}$ F. Yan, ${ }^{56}$ L. Yan, ${ }^{58 a, 58 c}$ W. B. Yan,${ }^{55,43}$ W. C. Yan, ${ }^{2}$ Y. H. Yan, ${ }^{20}$ H. J. Yang, ${ }^{38, \|}$ H. X. Yang, ${ }^{1}$ L. Yang, ${ }^{60}$ R. X. Yang, ${ }^{55,43}$ S. L. Yang, ${ }^{1,47}$ Y. H. Yang, ${ }^{33}$ Y. X. Yang, ${ }_{56}$ Yifan Yang, ${ }^{1,47}$ Z. Q. Yang, ${ }^{20}$ M. Ye, ${ }^{1,43}$ M. H. Ye, J. H. Yin, ${ }^{1}$ Z. Y. You, ${ }^{44}$ B. X. Yu, ${ }^{1,43,47}$ C. X. Yu, ${ }^{34}$ J. S. Yu, ${ }^{20}$ T. Yu, ${ }^{56}$

C. Z. Yuan, ${ }^{1,47}$ X. Q. Yuan, ${ }^{35, \dagger}$ Y. Yuan, ${ }^{1}$ A. Yuncu, ${ }^{46 b, f \uparrow}$ A. A. Zafar ${ }^{57}$ Y. Zeng, ${ }^{20}$ B. X. Zhang, ${ }^{1}$ B. Y. Zhang, ${ }^{1,43}$ C. C. Zhang, ${ }^{1}$ D. H. Zhang, ${ }^{1}$ H. H. Zhang, ${ }^{4}{ }^{4}$ H. Y. Zhang, ${ }^{1,43}$ J. Zhang, ${ }^{1,47}$ J. L. Zhang, ${ }^{61}$ J. Q. Zhang, ${ }^{4}$ J. W. Zhang, ${ }^{1,43,47}$ J. Y. Zhang, ${ }^{1}$ J. Z. Zhang, ${ }^{1,47}$ K. Zhang, ${ }^{1,47}$ L. Zhang, ${ }^{45}$ S. F. Zhang $\odot{ }^{33}$ T. J. Zhang, ${ }^{38, \|}$ X. Y. Zhang, ${ }^{37}$ Y. Zhang, ${ }^{55,43}$ Y. H. Zhang, ${ }^{1,43}$ Y. T. Zhang, ${ }^{55,43}$ Yang Zhang, ${ }^{1}$ Yao Zhang, ${ }^{1}$ Yi Zhang, ${ }^{9, \dagger}$ Yu Zhang, ${ }^{47}$ Z. H. Zhang, ${ }^{6}$ Z. P. Zhang, ${ }^{55}$ 
Z. Y. Zhang, ${ }^{60}$ G. Zhao, ${ }^{1}$ J. W. Zhao, ${ }^{1,43}$ J. Y. Zhao, ${ }^{1,47}$ J. Z. Zhao, ${ }^{1,43}$ Lei Zhao, ${ }^{55,43}$ Ling Zhao, ${ }^{1}$ M. G. Zhao, ${ }^{34}$ Q. Zhao, ${ }^{1}$ S. J. Zhao, ${ }^{63}$ T. C. Zhao, ${ }^{1}$ Y. B. Zhao, ${ }^{1,43}$ Z. G. Zhao, ${ }^{55,43}$ A. Zhemchugov, ${ }^{27, *}$ B. Zheng, ${ }^{56}$ J. P. Zheng, ${ }^{1,43}$ Y. Zheng, ${ }^{35, \dagger}$ Y. H. Zheng, ${ }^{47}$ B. Zhong, ${ }^{32}$ L. Zhou, ${ }^{1,43}$ L. P. Zhou, ${ }^{1,47}$ Q. Zhou, ${ }^{1,47}$ X. Zhou, ${ }^{60}$ X. K. Zhou, ${ }^{47}$ X. R. Zhou, ${ }^{55,43}$ Xiaoyu Zhou, ${ }^{20}$ Xu Zhou, ${ }^{20}$ A. N. Zhu, ${ }^{1,47}$ J. Zhu, ${ }^{34}$ J. Zhu, ${ }^{44}$ K. Zhu, ${ }^{1}$ K. J. Zhu, ${ }^{1,43,47}$ S. H. Zhu, ${ }^{54}$ W. J. Zhu, ${ }^{34}$ X. L. Zhu, ${ }^{45}$ Y. C. Zhu, ${ }^{55,43}$ Y. S. Zhu, ${ }^{1,47}$ Z. A. Zhu, ${ }^{1,47}$ J. Zhuang, ${ }^{1,43}$ B. S. Zou, ${ }^{1}$ and J. H. Zou ${ }^{1}$

\section{(BESIII Collaboration)}

${ }^{1}$ Institute of High Energy Physics, Beijing 100049, People's Republic of China

${ }^{2}$ Beihang University, Beijing 100191, People's Republic of China

${ }^{3}$ Beijing Institute of Petrochemical Technology, Beijing 102617, People's Republic of China

${ }^{4}$ Bochum Ruhr-University, D-44780 Bochum, Germany

${ }^{5}$ Carnegie Mellon University, Pittsburgh, Pennsylvania 15213, USA

${ }^{6}$ Central China Normal University, Wuhan 430079, People's Republic of China

${ }^{7}$ China Center of Advanced Science and Technology, Beijing 100190, People's Republic of China

${ }^{8}$ COMSATS University Islamabad, Lahore Campus, Defence Road,

Off Raiwind Road, 54000 Lahore, Pakistan

${ }^{9}$ Fudan University, Shanghai 200443, People's Republic of China

${ }^{10}$ G.I. Budker Institute of Nuclear Physics SB RAS (BINP), Novosibirsk 630090, Russia

${ }^{11}$ GSI Helmholtzcentre for Heavy Ion Research GmbH, D-64291 Darmstadt, Germany

${ }^{12}$ Guangxi Normal University, Guilin 541004, People's Republic of China

${ }^{13}$ Guangxi University, Nanning 530004, People's Republic of China

${ }^{14}$ Hangzhou Normal University, Hangzhou 310036, People's Republic of China

${ }^{15}$ Helmholtz Institute Mainz, Johann-Joachim-Becher-Weg 45, D-55099 Mainz, Germany

${ }^{16}$ Henan Normal University, Xinxiang 453007, People's Republic of China

${ }^{17}$ Henan University of Science and Technology, Luoyang 471003, People's Republic of China

${ }^{18}$ Huangshan College, Huangshan 245000, People's Republic of China

${ }^{19}$ Hunan Normal University, Changsha 410081, People's Republic of China

${ }^{20}$ Hunan University, Changsha 410082, People's Republic of China

${ }^{21}$ Indian Institute of Technology Madras, Chennai 600036, India

${ }^{22}$ Indiana University, Bloomington, Indiana 47405, USA

${ }^{23 a}$ INFN Laboratori Nazionali di Frascati, I-00044 Frascati, Italy

${ }^{23 \mathrm{~b}}$ INFN and University of Perugia, I-06100 Perugia, Italy

${ }^{24 \mathrm{a}}$ INFN Sezione di Ferrara, I-44122 Ferrara, Italy

${ }^{24 \mathrm{~b}}$ University of Ferrara, I-44122 Ferrara, Italy

${ }^{25}$ Institute of Physics and Technology, Peace Ave. 54B, Ulaanbaatar 13330, Mongolia

${ }^{26}$ Johannes Gutenberg University of Mainz, Johann-Joachim-Becher-Weg 45, D-55099 Mainz, Germany

${ }^{27}$ Joint Institute for Nuclear Research, 141980 Dubna, Moscow Region, Russia

${ }^{28}$ Justus-Liebig-Universitaet Giessen, II. Physikalisches Institut, Heinrich-Buff-Ring 16,

D-35392 Giessen, Germany

${ }^{29}$ KVI-CART, University of Groningen, NL-9747 AA Groningen, The Netherlands

${ }^{30}$ Lanzhou University, Lanzhou 730000, People's Republic of China

${ }^{31}$ Liaoning University, Shenyang 110036, People's Republic of China

${ }^{32}$ Nanjing Normal University, Nanjing 210023, People's Republic of China

${ }^{33}$ Nanjing University, Nanjing 210093, People's Republic of China

${ }^{34}$ Nankai University, Tianjin 300071, People's Republic of China

${ }^{35}$ Peking University, Beijing 100871, People's Republic of China

${ }^{36}$ Shandong Normal University, Jinan 250014, People's Republic of China

${ }^{37}$ Shandong University, Jinan 250100, People's Republic of China

${ }^{38}$ Shanghai Jiao Tong University, Shanghai 200240, People's Republic of China

${ }^{39}$ Shanxi University, Taiyuan 030006, People's Republic of China

${ }^{40}$ Sichuan University, Chengdu 610064, People's Republic of China

${ }^{41}$ Soochow University, Suzhou 215006, People's Republic of China

${ }^{42}$ Southeast University, Nanjing 211100, People's Republic of China

${ }^{43}$ State Key Laboratory of Particle Detection and Electronics, Beijing 100049, Hefei 230026,

People's Republic of China

${ }^{44}$ Sun Yat-Sen University, Guangzhou 510275, People's Republic of China

${ }^{45}$ Tsinghua University, Beijing 100084, People's Republic of China

${ }^{46 a}$ Ankara University, 06100 Tandogan, Ankara, Turkey

${ }^{46 \mathrm{~b}}$ Istanbul Bilgi University, 34060 Eyup, Istanbul, Turkey

${ }^{46 c}$ Uludag University, 16059 Bursa, Turkey 


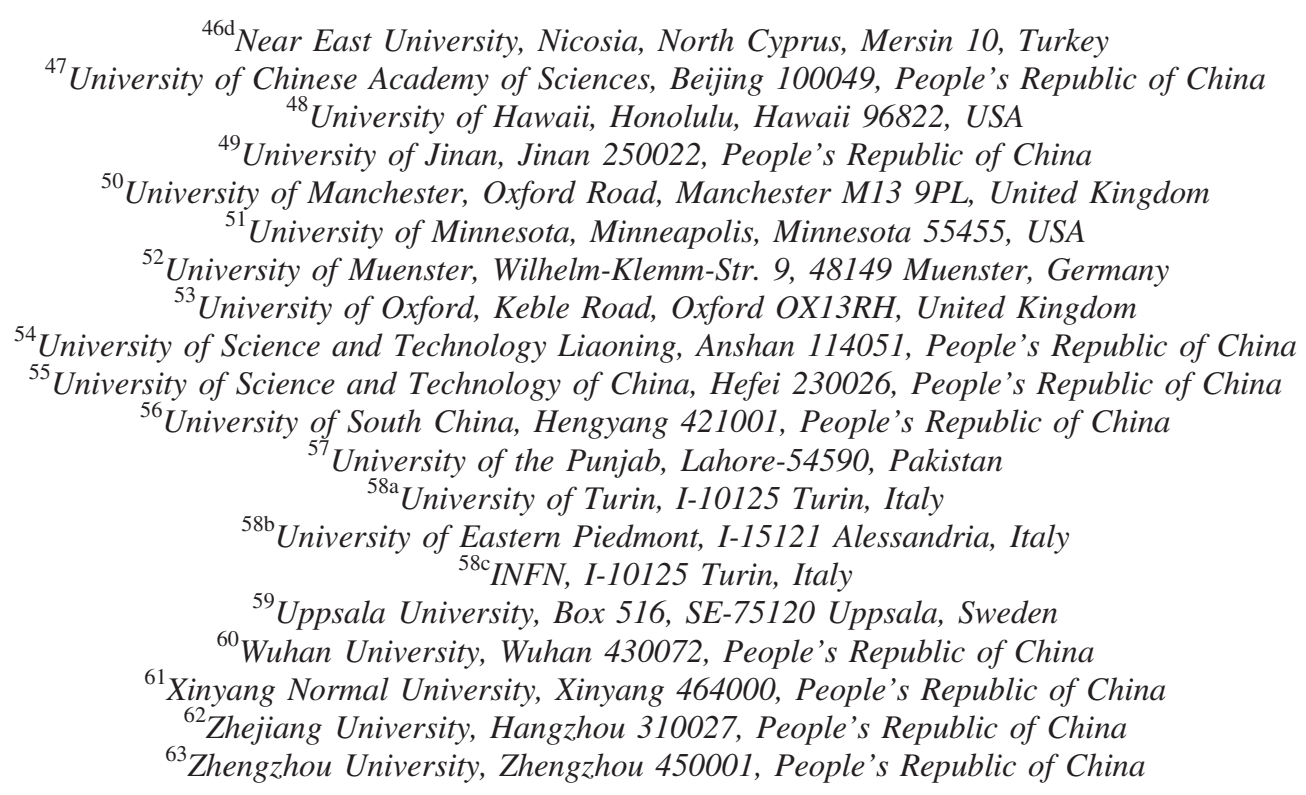

(Received 2 December 2019; accepted 21 January 2020; published 13 February 2020)

Using a $2.93 \mathrm{fb}^{-1}$ data sample of electron-positron collisions taken with the BESIII detector at a centerof-mass energy of $3.773 \mathrm{GeV}$, which corresponds to $(8296 \pm 31 \pm 64) \times 10^{3} D^{+} D^{-}$pairs, we search for the baryon and lepton number violating decays $D^{+} \rightarrow \bar{\Lambda}\left(\bar{\Sigma}^{0}\right) e^{+}$and $D^{+} \rightarrow \Lambda\left(\Sigma^{0}\right) e^{+}$. No obvious signals are found with the current statistics and upper limits on the branching fractions of these four decays are set at the level of $10^{-6}$ at $90 \%$ confidence level.

DOI: 10.1103/PhysRevD.101.031102

*Also at the Novosibirsk State University, Novosibirsk 630090, Russia.

${ }^{\dagger}$ Also at State Key Laboratory of Nuclear Physics and Technology, Peking University, Beijing 100871, People's Republic of China.

*Also at the Moscow Institute of Physics and Technology, Moscow 141700, Russia.

${ }^{\S}$ Also at the Functional Electronics Laboratory, Tomsk State University, Tomsk 634050, Russia.

"Also at Key Laboratory for Particle Physics, Astrophysics and Cosmology, Ministry of Education; Shanghai Key Laboratory for Particle Physics and Cosmology; Institute of Nuclear and Particle Physics, Shanghai 200240, People's Republic of China.

Also at Goethe University Frankfurt, 60323 Frankfurt am Main, Germany.

${ }^{* *}$ Also at Istanbul Arel University, 34295 Istanbul, Turkey.

${ }^{\dagger}$ Also at Key Laboratory of Nuclear Physics and Ion-beam Application (MOE) and Institute of Modern Physics, Fudan University, Shanghai 200443, People's Republic of China.

\#Also at Harvard University, Department of Physics, Cambridge, Massachusetts 02138, USA.

$\S$ Also at Government College Women University, Sialkot51310, Punjab, Pakistan.

"Al Also at the NRC "Kurchatov Institute", PNPI, 188300 Gatchina, Russia.

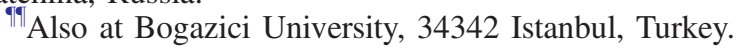

Published by the American Physical Society under the terms of the Creative Commons Attribution 4.0 International license. Further distribution of this work must maintain attribution to the author(s) and the published article's title, journal citation, and DOI. Funded by SCOAP ${ }^{3}$.

\section{INTRODUCTION}

In the standard model (SM), baryon number is conserved as a consequence of the $S U(2) \times U(1)$ and $S U(3)$ gauge symmetries. However, the fact that there is an excess of baryons over antibaryons in the Universe suggests the existence of baryon number violating (BNV) processes. Thus, the search for BNV processes can shed light on the evolution of the Universe. For decades, the decay of the proton, which is the lightest baryon, has been searched for, but no evidence for its decay has yet been found. An alternative probe is to look for the BNV decays of heavy mesons. Various SM extensions with BNV processes have been proposed [1-8]. Under dimension six operators, BNV processes can happen with $\Delta(B-L)=0$, where $\Delta(B-L)$ is the change in the difference between baryon and lepton numbers. In models including heavy gauge bosons $X$ with charge $\frac{4}{3}$ and gauge bosons $Y$ with charge $\frac{1}{3}$, one obtains the Feynman diagrams for BNV decays of $D$ mesons shown in Figs. 1(a) and 1(b). Another class of BNV operators is the class of dimension seven operators where $\Delta(B-L)=2$, as shown in the Feynman diagrams in Figs. 1(c) and 1(d). Reference [5] argues that the decay amplitudes of these two kinds of BNV processes may be comparable. A higher generation supersymmetry (SUSY) model predicts that the branching fraction (BF) of $D^{+} \rightarrow \bar{\Lambda} \ell^{+}$is no more than $10^{-29}$ [8] with the experimental limit of proton decay, where $\ell^{+}$represents $e^{+}$or $\mu^{+} . D^{+}$BNV decays 


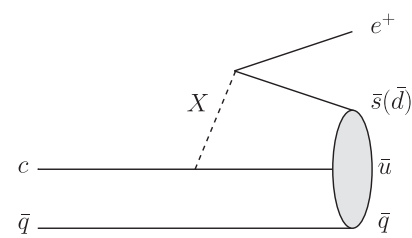

(a)

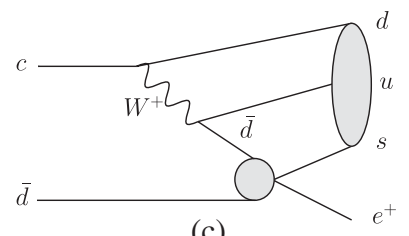

(c)

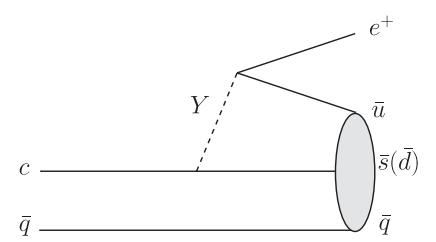

(b)

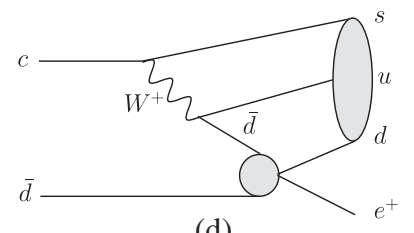

(d)
FIG. 1. Feynman diagrams for the BNV decays of $D$ mesons with $\Delta(B-L)$ equal to $0[(\mathrm{a})$ and (b)] and $2[(\mathrm{c})$ and (d)].

to the $\bar{\Sigma}^{0}$ baryon should have a $\mathrm{BF}$ at similar magnitude. Nevertheless, an experimental search for these BNV decays will probe new physics effects and test theoretical models beyond the SM.

Previously, CLEO [9] and BABAR [10] searched for BNV processes in $D$ and $B$ decays, and recently hyperon BNV decays were searched at CLAS [11], but no evidence was found. The upper limits (ULs) at $90 \%$ confidence level were set to be at the level of $10^{-5}-10^{-8}$. In this paper, by analyzing $2.93 \mathrm{fb}^{-1}$ of data taken at a center-of-mass energy of $\sqrt{s}=$ $3.773 \mathrm{GeV}$ with the BESIII detector, we report the first searches for the BNV decays $D^{+} \rightarrow \bar{\Lambda} e^{+}$and $D^{+} \rightarrow \bar{\Sigma}^{0} e^{+}$ with $\Delta(B-L)=0$, as well as $D^{+} \rightarrow \Lambda e^{+}$and $D^{+} \rightarrow \Sigma^{0} e^{+}$ with $\Delta(B-L)=2$. Throughout this paper, charge-conjugated channels are implied unless explicitly stated.

\section{THE BESIII EXPERIMENT AND DATA SAMPLE}

The BESIII detector is a magnetic spectrometer [12] located at the Beijing Electron Positron Collider [13]. The cylindrical core of the BESIII detector consists of a heliumbased multilayer drift chamber, a plastic scintillator timeof-flight system (TOF), and a CsI(Tl) electromagnetic calorimeter (EMC), which are all enclosed in a superconducting solenoidal magnet providing a $1.0 \mathrm{~T}$ magnetic field. The solenoid is supported by an octagonal flux-return yoke with resistive plate counter muon identifier modules interleaved with steel. The acceptance of charged particles and photons is $93 \%$ over $4 \pi$ solid angle. The charged particle momentum resolution at $1 \mathrm{GeV} / c$ is $0.5 \%$, and the $d E / d x$ resolution is $6 \%$ for the electrons from Bhabha scattering. The EMC measures photon energies with a resolution of $2.5 \%(5 \%)$ at $1 \mathrm{GeV}$ in the barrel (end-cap) region. The time resolution of the TOF barrel region is $68 \mathrm{ps}$, while that of the end cap is 110 ps.

Simulated samples of events produced with a GEANT4based [14] Monte Carlo (MC) package, which includes the geometric description of the BESIII detector and the detector response, are used to determine the detection efficiency and to estimate the backgrounds. The simulation includes the beam energy spread and initial state radiation (ISR) in the $e^{+} e^{-}$annihilations modeled with the generator KKMC [15]. The inclusive MC samples consist of the production of $D \bar{D}$ pairs including quantum coherence for all neutral $D$ modes, the non- $D \bar{D}$ decays of the $\psi(3770)$, the ISR production of the $J / \psi$ and $\psi(3686)$ states, and the continuum processes incorporated in KKMC [15]. The known decay modes are modeled with EVTGEN $[16,17]$ using BFs taken from the Particle Data Group (PDG) [18], and the remaining unknown decays of the charmonium states are simulated with LUNDCHARM $[19,20]$. Final state radiation from charged final state particles is incorporated with the PнотоS [21] package.

\section{EVENT SELECTION}

To avoid possible bias, a blind analysis technique is followed where the data are viewed only after the analysis procedure is fixed and validated with MC simulation. The BNV decays are searched for using all tracks reconstructed within the polar angle range $|\cos \theta|<0.93$ with respect to the beam axis. The $\Lambda$ and $\Sigma^{0}$ baryons are reconstructed via the $\Lambda \rightarrow p \pi^{-}$and $\Sigma^{0} \rightarrow \gamma \Lambda$ decays, respectively. Each track used to reconstruct a $\Lambda$ baryon is required to have a distance of closest approach to the interaction point (IP) along the beam axis of less than $20 \mathrm{~cm}$. Particle identification (PID) is applied to the charged tracks using information from the $d E / d x$ and TOF measurements. The confidence levels for pion, kaon, and proton hypotheses $\left(C L_{\pi}, C L_{K}\right.$, and $\left.C L_{p}\right)$ are calculated. The proton candidates are required to satisfy $C L_{p}>0.001, C L_{p}>C L_{\pi}$, and $C L_{p}>C L_{K}$, while no PID is required for the pion candidates. A vertex fit is performed to constrain the proton and pion tracks to a common vertex and the $\chi^{2}$ of the fit is required to be less than 100 . The distance between the IP and the $\Lambda$ decay vertex is required to be larger than 2 standard deviations of the vertex resolution, and the invariant mass of the $p \pi^{-}$combination is required to be within $(1.110,1.121) \mathrm{GeV} / c^{2}$.

Photons are selected from the isolated EMC showers whose energy lost in the TOF has been recovered. The shower must start within $700 \mathrm{~ns}$ of the event start time and is required to have an energy greater than 25 (50) $\mathrm{MeV}$ in the barrel (end-cap) region of the EMC. The minimum opening angle between the shower and any charged track has to be greater than $10^{\circ}$. To form a $\Sigma^{0}$ candidate, the invariant mass of the $\gamma p \pi^{-}$combination is required to be within $(1.173,1.200) \mathrm{GeV} / c^{2}$. Figure 2 shows the invariant mass distributions of the $\Lambda$ and $\Sigma^{0}$ candidates in the MC simulation.

The positron candidates are required to have a distance of closest approach to the IP of less than $1 \mathrm{~cm}$ in the tranverse plane and less than $10 \mathrm{~cm}$ along the beam axis. Positron PID is performed using $d E / d x$, TOF, and EMC information, with which the confidence levels for positron, pion, kaon, and proton hypotheses $\left(C L_{e}, C L_{\pi}, C L_{K}\right.$, 

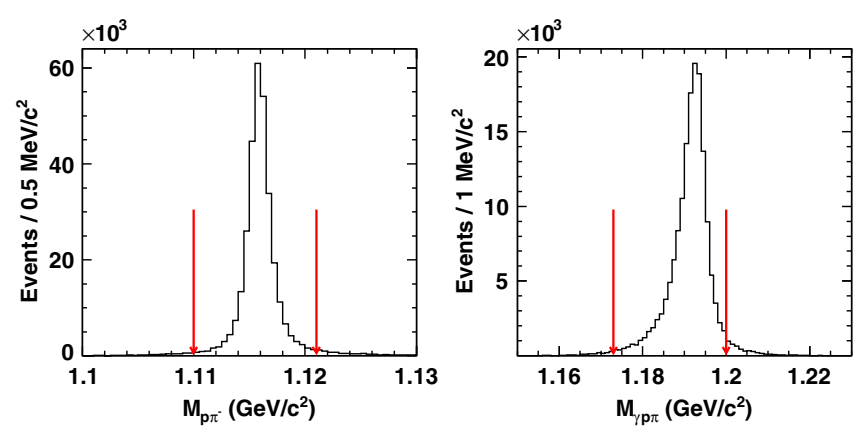

FIG. 2. The invariant mass distributions of the $\Lambda$ (left) and $\Sigma^{0}$ (right) candidates from the generated signal MC events, where the arrows give the mass windows.

and $C L_{p}$ ) are calculated. Positron candidates are required to satisfy $C L_{e}>0.001$ and

$$
\frac{C L_{e}}{C L_{e}+C L_{\pi}+C L_{K}+C L_{p}}>0.8 \text {. }
$$

In addition, the ratio of the energy deposited in the EMC by the positron over its momentum $(E / p)$ is required to be within $(0.8,1.2)$.

The BNV decays of the $D^{+}$mesons are identified using the energy difference $\Delta E=E_{D}-E_{\text {beam }}$ and the beam constrained mass $M_{\mathrm{BC}}=\sqrt{E_{\text {beam }}^{2}-p_{D}^{2}}$, where $E_{\text {beam }}$ is the beam energy, and $E_{D}$ and $p_{D}$ are the energy and momentum of the $D^{+}$candidate in the rest frame of the $e^{+} e^{-}$system. When multiple candidates for a specific signal mode are present, the one with $\Delta E$ nearest to 0 is retained. The $D^{+}$ candidate must satisfy $-0.023<\Delta E<0.022(-0.028<$ $\Delta E<0.024) \mathrm{GeV}$ for $D^{+} \rightarrow \Lambda e^{+}$and $D^{+} \rightarrow \bar{\Lambda} e^{+}$ $\left(D^{+} \rightarrow \Sigma^{0} e^{+}\right.$and $\left.D^{+} \rightarrow \bar{\Sigma}^{0} e^{+}\right)$, as shown in Fig. 3 .

Studies of MC samples show that there remain a few backgrounds coming from misreconstructed $\Lambda$, e.g., $D^{+} \rightarrow$ $\bar{K}^{0} e^{+} \nu_{e}$ and $D^{+} \rightarrow \bar{K}^{*}(892)^{0} e^{+} \nu_{e}$. However, most backgrounds are from processes other than $\psi(3770) \rightarrow D \bar{D}$ where a real $\Lambda(\bar{\Lambda})$ is produced. In this case, the reconstructed positron candidates are mainly from photon conversion, decay products of pions, muons, or kaons,
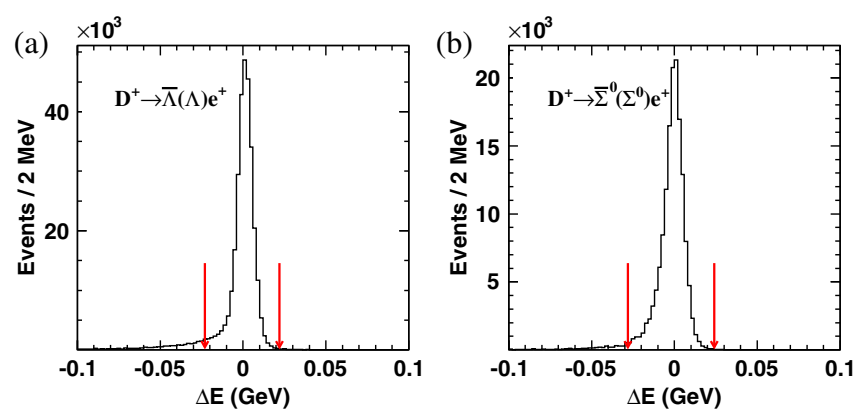

FIG. 3. The $\Delta E$ distributions from generated signal $\mathrm{MC}$ events for $D^{+} \rightarrow \bar{\Lambda}(\Lambda) e^{+}$(a) and $D^{+} \rightarrow \bar{\Sigma}^{0}\left(\Sigma^{0}\right) e^{+}$(b), where the arrows give the signal windows. as well as misidentification from other kinds of particles. Backgrounds produced due to photon conversion are identified using the technique introduced in Ref. [22]. An electron-positron pair is formed by looping over all electrons in the event. The electron with minimum angle relative to the positron candidate is chosen. Three variables, the minimum signed distance between the electron and positron in the $x y$ plane $\Delta_{x y}$, the polar angle of the direction of the conversion photon with respect to the vector from the IP to the common vertex of the electron-positron pair $\theta_{e g}$, and the distance between the common vertex of the electron-positron pair and the IP in the $x y$ plane $R_{x y}$, are defined. Events with $-2<\Delta_{x y}<1 \mathrm{~cm}, \cos \theta_{e g}>0.8$, and $R_{x y}>2$ are identified as background associated with photon conversions and are rejected. To suppress backgrounds from the $e^{+} e^{-} \rightarrow q \bar{q}$ process and charmonium decays which may contain a baryon-antibaryon pair, we require that no charged particle satisfies the proton PID criteria, except the proton from the BNV decay candidate.

Figure 4 shows the $M_{\mathrm{BC}}$ distributions of the accepted candidate events in data and inclusive MC samples. A maximum likelihood fit to the $M_{\mathrm{BC}}$ distribution is performed on each distribution of data to extract the number of signal events in each signal decay mode. In the fit, the signal is modeled by an MC-simulated shape convolved with a Gaussian to account for the resolution difference between data and MC simulation and the background is modeled by an ARGUS function [23], which has been found to be in agreement with inclusive MC samples. The end point of the ARGUS function is fixed at the beam energy and other parameters are determined from the fit. The data/MC difference in $M_{\mathrm{BC}}$ resolution is estimated using the topologically similar decays $D^{+} \rightarrow K_{S}^{0} \pi^{+}$and $D^{+} \rightarrow K_{S}^{0} \pi^{+} \pi^{0}$ for signal channels involving $\Lambda$ and $\Sigma^{0}$,

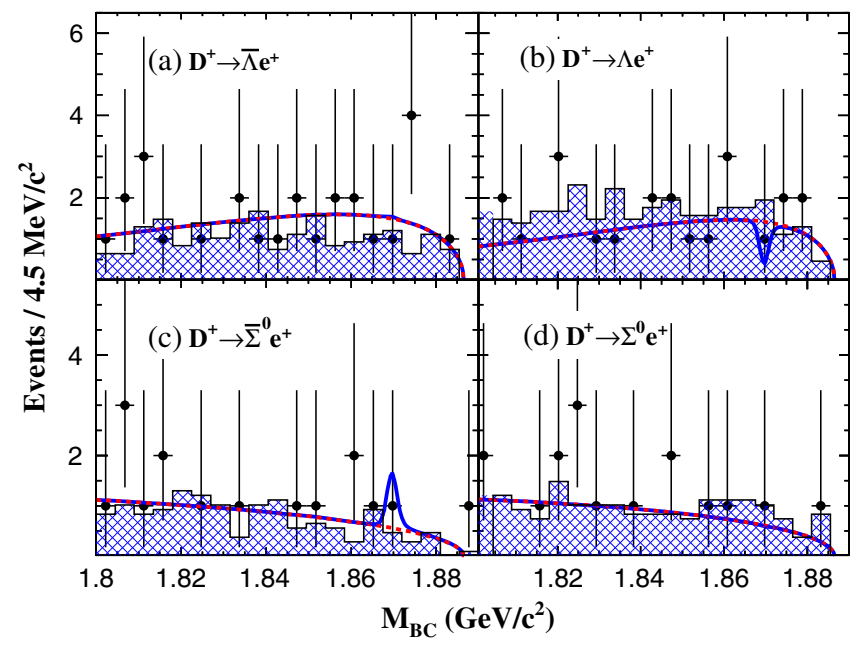

FIG. 4. Fits to the $M_{\mathrm{BC}}$ distributions of the accepted candidate events in data, where the dots with error bars are data, the solid curves are the best fits, and the red dashed curves are the background shapes. The blue hatched histograms are the MC-simulated backgrounds scaled to data according to the luminosity. 
respectively. The efficiencies of reconstructing the four signal decay modes are estimated using the signal MC samples, in which signal events are generated with unpolarized particles.

\section{SYSTEMATIC UNCERTAINTIES}

The systematic uncertainties in the searches for these BNV decays excluding those involved in the $M_{\mathrm{BC}}$ fit are summarized in Table I. The total number of $D^{+} D^{-}$pairs in the data set was previously measured in Ref. [24] with an uncertainty of $0.9 \%$. The uncertainties in the tracking and PID efficiencies of the positron are studied using $e^{+} e^{-} \rightarrow$ $\gamma e^{+} e^{-}$events. To account for the difference in kinematics between the positrons in the control sample and the signal decays, the tracking and PID efficiencies are estimated by weighting the efficiencies extracted from the control sample in two-dimensional (momentum and $\cos \theta$ ) distributions. The differences of the weighted efficiencies between data and MC simulation, which are $0.3 \%$ for tracking and $1.0 \%$ for PID, are taken as the associated systematic uncertainties. The uncertainty in the reconstruction efficiency of $\Lambda(\bar{\Lambda})$ was previously studied in Ref. [25] using $J / \psi \rightarrow \Lambda \bar{\Lambda} \pi^{+} \pi^{-}$events. The momentum-weighted difference of $\Lambda(\bar{\Lambda})$ reconstruction efficiencies between data and MC simulation is $1.5 \%$, which is assigned as an uncertainty of the $\Lambda(\bar{\Lambda})$ reconstruction. This includes the uncertainties in the tracking efficiencies of the pion and proton, the PID efficiency of the proton, the decay length requirement, and the mass window. For decays involving the $\Sigma^{0}\left(\bar{\Sigma}^{0}\right)$ baryon, the uncertainty in the photon reconstruction efficiency is taken to be $1.0 \%$ according to the previous study using $J / \psi \rightarrow \pi^{+} \pi^{-} \pi^{0}$ events [26]. The uncertainty in the requirement of the mass window of the $\Sigma^{0}\left(\bar{\Sigma}^{0}\right)$ baryon is studied with $J / \psi \rightarrow p K^{-} \bar{\Sigma}^{0}+$ c.c. events, and is found to be negligible. The uncertainties in the $\Delta E$ requirement are estimated by smearing the $\mathrm{MC}$ simulated $\Delta E$ distributions with Gaussian functions accounting for the resolution difference between data

TABLE I. The systematic uncertainties excluding those involved in the $M_{\mathrm{BC}}$ fit (in \%) for the four signal channels.

\begin{tabular}{lccrr}
\hline \hline Source & $\bar{\Lambda} e^{+}$ & $\Lambda e^{+}$ & $\bar{\Sigma}^{0} e^{+}$ & $\Sigma^{0} e^{+}$ \\
\hline$N_{D^{+}}^{\text {tot }} D^{-}$ & 0.9 & 0.9 & 0.9 & 0.9 \\
$\Delta E$ cut & 0.6 & 0.6 & 0.9 & 0.9 \\
$\Lambda(\bar{\Lambda})$ reconstruction & 1.5 & 1.5 & 1.5 & 1.5 \\
$\Sigma^{0}\left(\overline{\Sigma^{0}}\right)$ mass window & & & $<0.1$ & $<0.1$ \\
$e^{+}$tracking & 0.3 & 0.3 & 0.3 & 0.3 \\
$e^{+}$PID & 1.0 & 1.0 & 1.0 & 1.0 \\
$\gamma$ reconstruction & & & 1.0 & 1.0 \\
MC statistics & 0.3 & 0.4 & 0.4 & 0.4 \\
No extra (anti-)proton & 0.3 & 0.3 & 0.3 & 0.3 \\
Photon conversion veto & 0.5 & 0.5 & 0.5 & 0.5 \\
Quoted BF(s) & 0.8 & 0.8 & 0.9 & 0.9 \\
Total & 2.4 & 2.4 & 2.7 & 2.7 \\
\hline \hline
\end{tabular}

and $\mathrm{MC}$ simulation. The efficiency changes after smearing are taken to be the associated systematic uncertainties, which are $0.6 \%, 0.6 \%, 0.9 \%$, and $0.9 \%$ for $D^{+} \rightarrow \bar{\Lambda} e^{+}$, $D^{+} \rightarrow \Lambda e^{+}, D^{+} \rightarrow \bar{\Sigma}^{0} e^{+}$, and $D^{+} \rightarrow \Sigma^{0} e^{+}$, respectively. To study the uncertainty in the photon conversion veto, we separately examine the data-MC difference in finding an extra electron in the system recoiling against the $D^{+}$meson and that for the $R_{x y}, \Delta_{x y}$ and $\cos \theta_{e g}$ requirement. The former is studied using the selected $D^{+} \rightarrow K_{S}^{0} \pi^{+}$vs $D^{-} \rightarrow$ anything sample, and the latter with $J / \psi \rightarrow$ $\pi^{+} \pi^{-} \pi^{0}, \pi^{0} \rightarrow \gamma e^{+} e^{-}$events. Combining these two studies, we assign $0.5 \%$ for the uncertainty in the photon conversion veto for the four signal decay modes. The uncertainty in the requirement of no extra proton (antiproton) is studied using the selected $D^{+} \rightarrow K_{S}^{0} \pi^{+}$vs $D^{-} \rightarrow$ anything sample. The difference of the acceptance rates of no additional proton (antiproton) between data and MC simulation, $0.3 \%$, is assigned as the associated uncertainty. We take $0.8 \%$ as the uncertainty in the BF of $\Lambda \rightarrow p \pi^{-}$quoted from the PDG [18] and $0.5 \%$ for $\Sigma^{0} \rightarrow \gamma \Lambda$ by referring to a theoretical value of the $\mathrm{BF}$ of $\Sigma^{0} \rightarrow \Lambda e^{+} e^{-}$[27]. In total, the uncertainties of the quoted $\mathrm{BFs}$ are $0.8 \%$ for $D^{+} \rightarrow$ $\bar{\Lambda}(\Lambda) e^{+}$and $0.9 \%$ for $D^{+} \rightarrow \bar{\Sigma}^{0}\left(\Sigma^{0}\right) e^{+}$. The limited MC statistics is also taken into account as a source of systematic uncertainty. The total systematic uncertainties are obtained by adding these uncertainties in quadrature.

\section{UPPER LIMIT ESTIMATION}

Since no significant signals are observed, we set the ULs on the BFs at $90 \%$ confidence level for the four signal decay modes. This is done by scanning the ratio of the likelihood value given the number of signal events and the maximum likelihood value $\left[\lambda\left(N_{\text {sig }}\right)\right]$ in the $M_{\mathrm{BC}}$ fit. The likelihood ratio distribution is then convoluted with a Gaussian function with corresponding width to incorporate the systematic

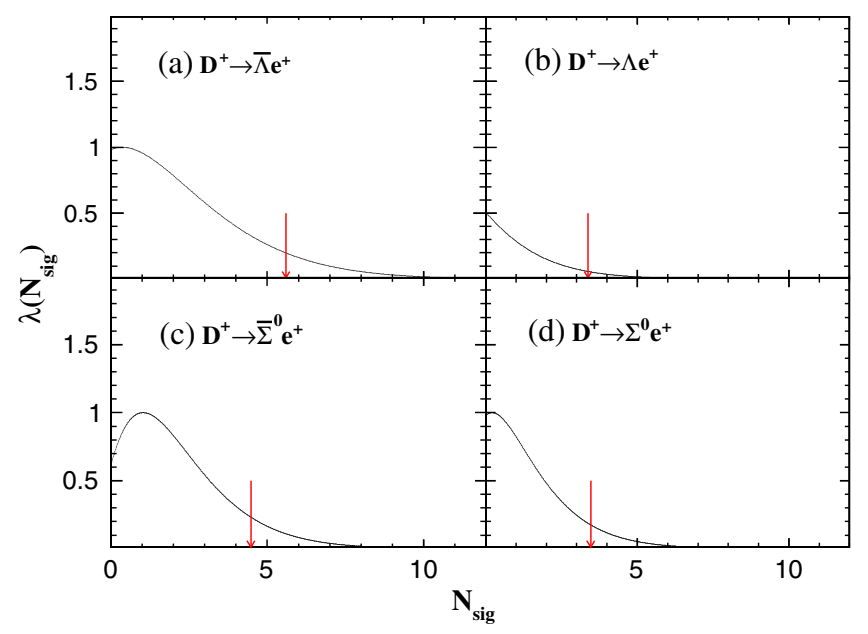

FIG. 5. The likelihood ratio distributions with respect to the number of signal events, where the red arrows give the upper limits at $90 \%$ confidence level. 
TABLE II. The ULs on the number of signal events at $90 \%$ confidence level, the detection efficiencies not including the BFs of the secondary decays, and the corresponding ULs on the BFs for the four signal decay modes, where the systematic uncertainties have been included.

\begin{tabular}{lccc}
\hline \hline Mode & $N_{\text {sig }}^{\mathrm{UL}}$ & $\varepsilon(\%)$ & $\mathcal{B}^{\mathrm{UL}}$ \\
\hline$\Lambda e^{+}$ & 5.6 & $31.11 \pm 0.14$ & $1.1 \times 10^{-6}$ \\
$\bar{\Lambda} e^{+}$ & 3.4 & $31.18 \pm 0.10$ & $6.5 \times 10^{-7}$ \\
$\Sigma^{0} e^{+}$ & 4.5 & $16.31 \pm 0.07$ & $1.7 \times 10^{-6}$ \\
$\bar{\Sigma}^{0} e^{+}$ & 3.5 & $16.40 \pm 0.07$ & $1.3 \times 10^{-6}$ \\
\hline \hline
\end{tabular}

uncertainties. The ULs on the number of signal events at $90 \%$ confidence level $\left(N_{\text {sig }}^{\mathrm{UL}}\right)$ are extracted by integrating over the physics region and finding the solution of

$$
\int_{0}^{N_{\text {sig }}^{\mathrm{UL}}} N_{\text {sample }} d N_{\text {sig }} / \int_{0}^{\infty} N_{\text {sample }} d N_{\text {sig }}=90 \%,
$$

where $N_{\text {sample }} d N_{\text {sig }}$ is the number of samples with the signal events between $N_{\text {sig }}$ and $N_{\text {sig }}+d N_{\text {sig. }}$. In addition, to account for the uncertainties introduced in the fitting method, we vary the signal shape, background shape and fitting range and keep the maximum $N_{\text {sig }}^{\mathrm{UL}}$ given for each signal decay. To be specific, the signal shape is varied by changing the width of the convoluted Gaussian according to its uncertainty; the end point of the ARGUS function is altered from $1.8865 \mathrm{GeV} / c^{2}$ to 1.8864 and $1.8866 \mathrm{GeV} / c^{2}$; and the fit is performed within three different regions: $(1.80,1.89),(1.81,1.89)$, and $(1.82,1.89) \mathrm{GeV} / c^{2}$. Figure 5 shows the likelihood ratio distributions with respect to the number of signal events for the four signal decays. For each signal decay mode, the UL on the $\mathrm{BF}$ is calculated as

$$
\mathcal{B}^{\mathrm{UL}}=N_{\text {sig }}^{\mathrm{UL}} /\left(2 \times N_{D^{+} D^{-}}^{\mathrm{tot}} \times \varepsilon \times \mathcal{B}_{\Lambda, \Sigma^{0}}\right),
$$

where $N_{D^{+} D^{-}}^{\text {tot }}$ is the total number of $D^{+} D^{-}$pairs which was measured to be $(8296 \pm 31 \pm 64) \times 10^{3}$ [24], $\varepsilon$ is the signal efficiency and $\mathcal{B}_{\Lambda, \Sigma^{0}}$ represents the BFs of the secondary decays used to reconstruct $\Lambda$ and $\Sigma^{0}$. Table II summarizes the ULs on the numbers of signal events in data, the signal efficiencies and the corresponding ULs on the BFs for the four signal decay modes.

\section{SUMMARY}

In summary, using $2.93 \mathrm{fb}^{-1}$ of data taken at $\sqrt{s}=$ $3.773 \mathrm{GeV}$ with the BESIII detector, we have searched for the BNV decays $D^{+} \rightarrow \bar{\Lambda} e^{+}, D^{+} \rightarrow \bar{\Sigma}^{0} e^{+}, D^{+} \rightarrow \Lambda e^{+}$, and $D^{+} \rightarrow \Sigma^{0} e^{+}$for the first time with the assumption of no preferred polarization of the final products. No obvious signals are found, and the ULs on the BFs of these decays are set at 90\% confidence level, as shown in Table II. Our limits are far above the prediction of the higher generation model [8].

\section{ACKNOWLEDGMENTS}

The BESIII collaboration thanks the staff of BEPCII and the IHEP computing center for their strong support. This work is supported in part by National Key Basic Research Program of China under Contract No. 2015CB856700; National Natural Science Foundation of China (NSFC) under Contracts No. 11625523, No. 11635010, and No. 11735014; National Natural Science Foundation of China (NSFC) under Contract No. 11835012; the Chinese Academy of Sciences (CAS) Large-Scale Scientific Facility Program; Joint Large-Scale Scientific Facility Funds of the NSFC and CAS under Contracts No. U1532257, No. U1532258, No. U1732263, and No. U1832207; CAS Key Research Program of Frontier Sciences under Contracts No. QYZDJ-SSW-SLH003 and No. QYZDJSSW-SLH040; 100 Talents Program of CAS; Institute of Nuclear and Particle Physics (INPAC) and Shanghai Key Laboratory for Particle Physics and Cosmology; German Research Foundation DFG under Contract No. Collaborative Research Center CRC 1044; Istituto Nazionale di Fisica Nucleare, Italy; Koninklijke Nederlandse Akademie van Wetenschappen (KNAW) under Contract No. 530-4CDP03; Ministry of Development of Turkey under Contract No. DPT2006K120470; National Science and Technology fund; The Knut and Alice Wallenberg Foundation (Sweden) under Contract No. 2016.0157; The Swedish Research Council; U.S. Department of Energy under Contracts No. DE-FG0205ER41374, No. DE-SC-0010118, and No. DE-SC0012069; University of Groningen (RuG) and the Helmholtzzentrum fuer Schwerionenforschung $\mathrm{GmbH}$ (GSI), Darmstadt.
[1] S. Weinberg, Phys. Rev. Lett. 43, 1566 (1979).

[2] S. Weinberg, Phys. Rev. D 22, 1694 (1980).

[3] F. Wilczek and A. Zee, Phys. Rev. Lett. 43, 1571 (1979).

[4] H. A. Weldon and A. Zee, Nucl. Phys. 173, 269 (1980).
[5] F. Wilczek and A. Zee, Phys. Lett. B 88, 311 (1979).

[6] R. N. Mohapatra and R. E. Marshak, Phys. Lett. B 94, 183 (1980).

[7] K. Biswal, L. Maharana, and S. P. Misra, Phys. Rev. D 25, 266 (1982). 
[8] W. S. Hou, M. Nagashima, and A. Soddu, Phys. Rev. D 72, 095001 (2005).

[9] R. Rubin et al. (CLEO Collaboration), Phys. Rev. D 79, 097101 (2009).

[10] P. del Amo Sanzhez et al. (BABAR Collaboration), Phys. Rev. D 83, 091101 (2011).

[11] M.E. McCracken et al., Phys. Rev. D 92, 072002 (2015).

[12] M. Ablikim et al. (BESIII Collaboration), Nucl. Instrum. Methods Phys. Res., Sect. A 614, 345 (2010).

[13] C. H. Yu et al., Proceedings of IPAC2016, Busan, Korea (2016), https://doi.org/10.18429/JACoW-IPAC2016-TUYA01.

[14] S. Agostinelli et al. (GEANT4 Collaboration), Nucl. Instrum. Methods Phys. Res., Sect. A 506, 250 (2003).

[15] S. Jadach, B. F. L. Ward, and Z. Was, Phys. Rev. D 63, 113009 (2001); Comput. Phys. Commun. 130, 260 (2000).

[16] D. J. Lange, Nucl. Instrum. Methods Phys. Res., Sect. A 462, 152 (2001).
[17] R. G. Ping, Chin. Phys. C 32, 599 (2008).

[18] M. Tanabashi et al. (Particle Data Group), Phys. Rev. D 98, 030001 (2018).

[19] J. C. Chen, G. S. Huang, X. R. Qi, D. H. Zhang, and Y. S. Zhu, Phys. Rev. D 62, 034003 (2000).

[20] R. L. Yang, R. G. Ping, and H. Chen, Chin. Phys. Lett. 31, 061301 (2014).

[21] E. Richter-Was, Phys. Lett. B 303, 163 (1993).

[22] Z. R. Xu and K. L. He, Chin. Phys. C 36, 742 (2012).

[23] H. Albrecht et al. (ARGUS Collaboration), Phys. Lett. B 241, 278 (1990).

[24] M. Ablikim et al. (BESIII Collaboration), Chin. Phys. C 42, 083001 (2018).

[25] M. Ablikim et al. (BESIII Collaboration), Phys. Rev. D 86, 052004 (2012).

[26] M. Ablikim et al. (BESIII Collaboration), Phys. Rev. D 83, 112005 (2011).

[27] G. Feinberg, Phys. Rev. 109, 1019 (1958). 